\title{
The ZEPLIN-III Anti-Coincidence Veto Detector
}

\author{
D.Yu. Akimov, ${ }^{1}$ H.M. Araújo, ${ }^{2}$ E.J. Barnes,${ }^{3}$ V.A. Belov,${ }^{1}$ A.A. Burenkov,${ }^{1}$ V. Chepel,${ }^{4}$ A. Currie, ${ }^{2}$ B. Edwards,${ }^{5}$ \\ V. Francis, ${ }^{5}$ C. Ghag ${ }^{*},{ }^{3}$ A. Hollingsworth, ${ }^{3}$ M. Horn, ${ }^{2}$ G.E. Kalmus, ${ }^{5}$ A.S. Kobyakin, ${ }^{1}$ A.G. Kovalenko, ${ }^{1}$ \\ V.N. Lebedenko, ${ }^{2}$ A. Lindote,${ }^{4}{ }^{5}$ M.I. Lopes, ${ }^{4}$ R. Lüscher,${ }^{5}$ K. Lyons,${ }^{2}$ P. Majewski, ${ }^{5}$ A.St J. Murphy, ${ }^{3}$ F. Neves, ${ }^{4,2}$ \\ S.M. Paling, ${ }^{5}$ J. Pinto da Cunha, ${ }^{4}$ R. Preece, ${ }^{5}$ J.J. Quenby, ${ }^{2}$ L. Reichhart, ${ }^{3}$ P.R. Scovell, ${ }^{3}$ V.N. Solovov, ${ }^{4}$ \\ N.J.T. Smith, ${ }^{5}$ P.F. Smith, ${ }^{5}$ V.N. Stekhanov, ${ }^{1}$ T.J. Sumner, ${ }^{2}$ R. Taylor, ${ }^{3}$ C. Thorne, ${ }^{2}$ and R.J. Walker ${ }^{2}$ \\ ${ }^{1}$ Institute for Theoretical and Experimental Physics, Moscow, Russia \\ ${ }^{2}$ Blackett Laboratory, Imperial College London, UK \\ ${ }^{3}$ School of Physics \& Astronomy, University of Edinburgh, UK \\ ${ }^{4}$ LIP-Coimbra $\&$ Department of Physics of the University of Coimbra, Portugal \\ ${ }^{5}$ Particle Physics Department, Rutherford Appleton Laboratory, Chilton, UK
}

(Dated: May 29, 2022)

\begin{abstract}
The design, optimisation and construction of an anti-coincidence veto detector to complement the ZEPLIN-III direct dark matter search instrument is described. One tonne of plastic scintillator is arranged into 52 bars individually read out by photomultipliers and coupled to a gadoliniumloaded passive polypropylene shield. Particular attention has been paid to radiological content. The overall aim has been to achieve a veto detector of low threshold and high efficiency without the creation of additional background in ZEPLIN-III, all at a reasonable cost. Extensive experimental measurements of the components have been made, including radioactivity levels and performance characteristics. These have been used to inform a complete end-to-end Monte Carlo simulation that has then been used to calculate the expected performance of the new instrument, both operating alone and as an anti-coincidence detector for ZEPLIN-III. The veto device will be capable of rejecting over $65 \%$ of coincident nuclear recoil events from neutron background in the energy range of interest in ZEPLIN-III. This will reduce the background in ZEPLIN-III from $\simeq 0.4$ to $\simeq 0.14$ events per year in the WIMP acceptance region, a significant factor in the event of a non-zero observation. Furthermore, in addition to providing valuable diagnostic capabilities, the veto is capable of tagging over $15 \%$ for $\gamma$-ray rejection, all whilst contributing no significant additional background. In conjunction with the replacement of the internal ZEPLIN-III photomultiplier array, the new veto is expected to improve significantly the sensitivity of the ZEPLIN-III instrument to dark matter, allowing spin independent WIMP-nucleon cross sections below $10^{-8} \mathrm{pb}$ to be probed.
\end{abstract}

PACS numbers: 14.80.Ly; 21.60.Ka; 29.40.Mc; 95.35.+d

\section{INTRODUCTION}

ZEPLIN-III [1, 2] is a two-phase (liquid/gas) xenon detector developed to observe low energy nuclear recoils resulting from the elastic scattering of galactic weakly interacting massive particles (WIMPs) [3]. A key feature of ZEPLIN-III is the ability to discriminate between incident particle species for each event by recording both vacuum ultraviolet scintillation light and electroluminescence from ionisation of the xenon target [4, 5]. The ratio in signal strength from these channels differs for electron and for nuclear recoil interactions, allowing the efficient rejection of most background events. However, in the absence of an electromagnetic interaction, weak and strong elastic scattering events appear identical, making the rejection of single scatter background neutron events difficult [6 6 8]. Additionally, even with good discrimination, for large exposures a small fraction of electron-recoil events can be misidentified as nuclear recoils. With predicted WIMP event rates of less than

*corresponding author: c.ghag@ed.ac.uk
0.1 events $/ \mathrm{kg} /$ day, and with energy depositions of the order of $10 \mathrm{keV}$, dark matter detectors such as ZEPLINIII must reduce the number of background events, especially due to neutrons, by a very large factor. Typically this is achieved through the use of high purity components of low radionuclide content, hydrocarbon and lead shielding external to the detector to attenuate and moderate neutrons and $\gamma$-rays originating in the local environment, as well as the operation of the detector at a site with large rock overburden to reduce the cosmic muon flux. Having been manufactured from low background components such as oxygen free copper, shielded with $30 \mathrm{~cm}$ of high density polypropylene and lead, and situated at the Boulby Underground Laboratory, UK, the background event rate of ZEPLIN-III is already greatly reduced. Sensitivity to spin-independent WIMP-nucleon cross sections as low as $8.1 \times 10^{-8}$ pb for a WIMP mass of $55 \mathrm{GeV} / \mathrm{c}^{2}$ has been demonstrated [9], as well as a spin-dependent WIMP-neutron cross section as low as $1.8 \times 10^{-2} \mathrm{pb}$ [10]. However, to improve the sensitivity of the device further, ZEPLIN-III is being retrofitted with an active veto detector. Since WIMPs are highly unlikely to interact within a detector system twice, any event doing so may be discarded. Following this upgrade, the predicted combined background event rate in ZEPLIN- 
III translates into much less than one count within the WIMP acceptance region for a one year dataset. The implementation of this veto system, in conjunction with new custom-built ultra-low background photomultiplier tubes (PMTs), will allow ZEPLIN-III to probe spin independent WIMP-nucleon cross sections below the $10^{-8} \mathrm{pb}$ level.

Experimental measurements have been made of the individual performance and radiological content of all components. These results have been fed into an endto-end Monte Carlo simulation of the ZEPLIN-III instrument [2], coupled with the entire veto detector array located in the Boulby mine, to evaluate the overall performance. An iterative process has been followed in which, with the geometry of the scintillator defined, alternative additional components and light collection efficiency were evaluated, informed by further experimental data where necessary.

In the following sections we first describe the general design of the veto. We then provide details of the measurements of the radioactivity content of the components, the performance of components, and the optimisation process. Finally we provide results from the evaluation of the predicted overall performance of the veto.

\section{OVERALL DESIGN}

Figures 1 and 2 depict schematically the veto system, while Table I presents key parameters of the overall system and its components. It consists of 52 individual bars of plastic scintillator arranged so as to provide $>3 \pi$ sr coverage around ZEPLIN-III. Thirty-two bars are $100 \mathrm{~cm}$ long, $15 \mathrm{~cm}$ thick parallelepipeds with trapezoidal cross section, that form a closed ring of outer diameter $160 \mathrm{~cm}$ (the 'barrel'). These are arranged to stand on a continuous $30 \mathrm{~cm}$ thick base piece of passive polypropylene shielding. A single 3-inch PMT is optically coupled to one end of each bar. PMTs are a likely significant source of background radioactivity. As such they are positioned on the lower face of the scintillator bars, in recessed holes in the base piece, thus keeping them farther from the ZEPLIN-III xenon target.

Between the scintillator and the ZEPLIN-III instrument are positioned 32 pieces of $15 \mathrm{~cm}$ thick gadolinium (Gd) loaded polypropylene forming a barrel of outer diameter $130 \mathrm{~cm}$. The scintillator and polypropylene barrels are rotated with respect to one another to ensure no line-of-sight gaps to the detector. Polypropylene has been chosen because of its high hydrogen content, machinebility, and low radioactivity [11]. For ease of manufacture the gadolinium is added to the polypropylene in the form of $10 \mu \mathrm{m} \mathrm{Gd}{ }_{2} \mathrm{O}_{3}$ powder mixed in to Rutherford Type 71 epoxy [12]. This mixture is set within $2 \mathrm{~mm}$ wide, $10 \mathrm{~mm}$ pitch vertical slots throughout the polypropylene. Monte Carlo simulations and experimental tests indicate that for neutron capture there is negligible difference between a uniform distribution and a discrete pattern, provided

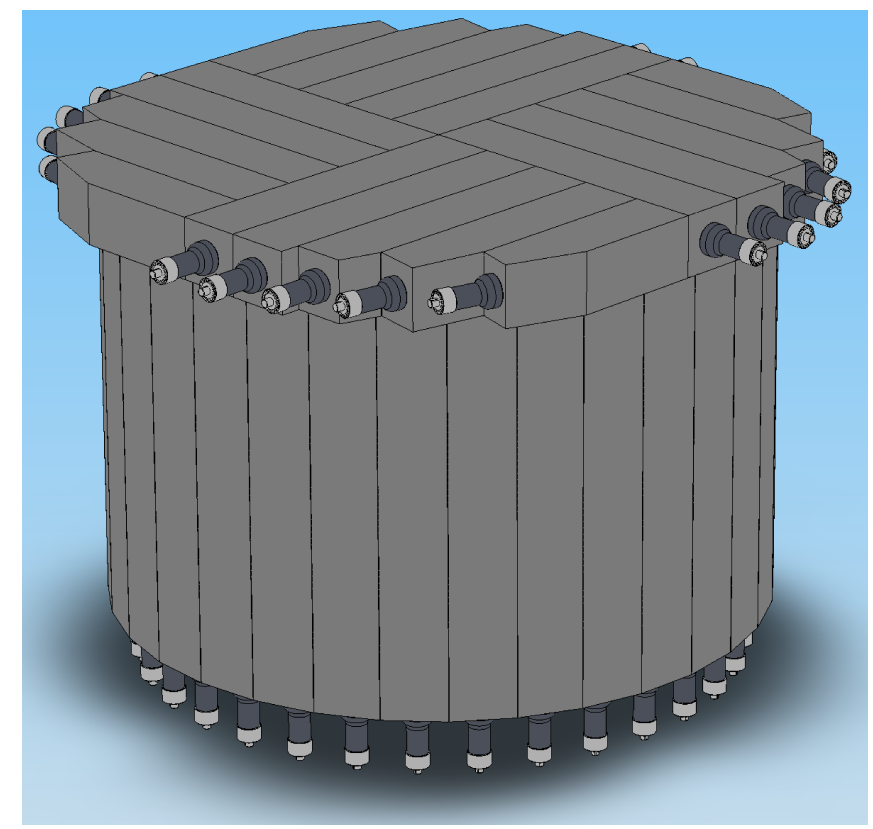

FIG. 1: Three dimensional rendering of the ZEPLIN-III veto detector, showing only the plastic scintillator bars with attached PMTs. The diameter of the barrel is $160 \mathrm{~cm}$

that the pitch of the slots does not exceed $\sim 15 \mathrm{~mm}$ (see section $\mathrm{VC}$ below). The polypropylene and the scintillator parallelepiped bars are secured together to provide mechanical stability. Monte Carlo simulations have also been employed to confirm that mm-sized voids between or within attached standing scintillator modules result in neither a significant loss in neutron detection efficiency nor increased exposure to background for ZEPLIN-III (see section VE below).

A $15 \mathrm{~cm}$ thick, $115 \mathrm{~cm}$ diameter disc of gadoliniumloaded polypropylene forms a roof piece, with the remaining 20 plastic scintillator bars resting on it to form a roof that extends over the full diameter of the barrel modules, as shown in Figure 1. A single PMT is optically coupled to the outer end of each roof bar, again maintaining maximum distance between veto PMTs and the xenon target. The combined thickness of the passive and active hydrocarbon shielding is $30 \mathrm{~cm}$, the same used in the first ZEPLIN-III science run [9]. The veto system is itself enclosed within the existing $20 \mathrm{~cm}$ thick Pb shielding of the ZEPLIN-III instrument (see Figure 2).

The geometry described above provides active rejection of background as follows. Firstly, neutrons entering the hydrocarbon shielding are efficiently moderated to thermal energies, mostly via $\mathrm{H}$ scattering, and undergo radiative capture predominantly on ${ }^{157} \mathrm{Gd}$ (natural abundance $15.7 \%$ ) which has an extraordinarily high capture cross section of $2.4 \times 10^{5}$ barns [13]. The level of gadolinium loading required was explored and is described in section $\mathrm{VC}$, where a value of at least $0.5 \%$ by weight, averaged over the polypropylene, is determined. The neutron capture is accompanied with the emission 


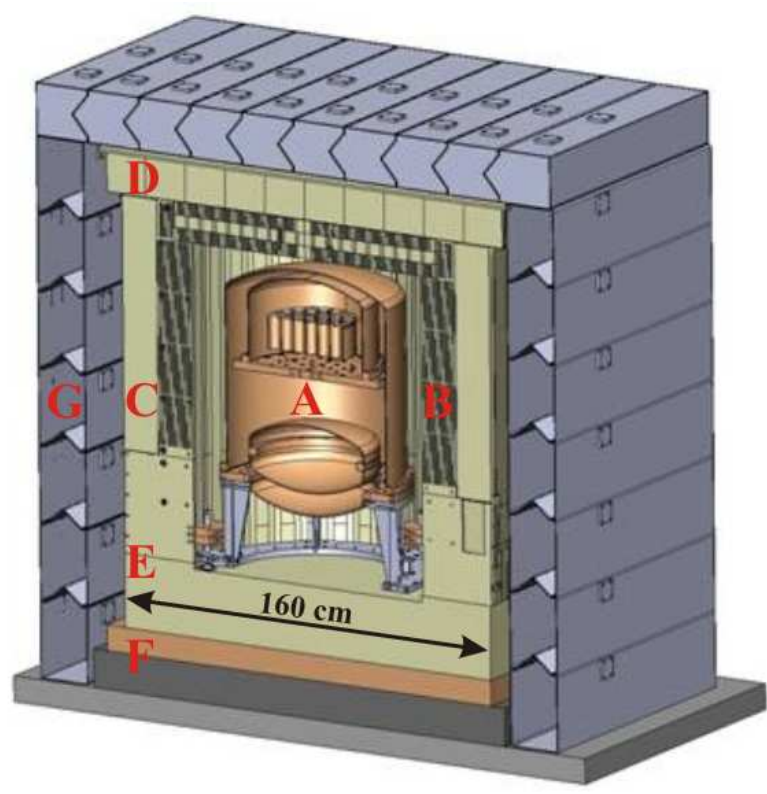

FIG. 2: Cross-sectional view of the ZEPLIN-III experiment in its second science run configuration. In the centre is the ZEPLIN-III detector (labelled A) showing the copper vacuum vessel enclosing the xenon target and the LN2 vessel below it. Forming a barrel around ZEPLIN-III are the 32 Gd-loaded polypropylene pieces and roof plug (labelled B and hatched). Surrounding these are the active scintillator modules (C) with PMTs housed in cups and recessed into the lower polypropylene structure. The 20 roof modules of scintillator (D) rest on the roof plug. The lower polypropylene structure (E) contains no Gd and rests on a copper and a lead base (F). Finally, the lead castle $(\mathrm{G})$ envelopes the entire assembly.

of typically $3-4 \gamma$-rays of energy totalling $\sim 8 \mathrm{MeV}$, and at a loading of $0.5 \%$ these are emitted with a mean delay of $35 \mu \mathrm{s}$ from the time the neutron enters the loaded material. This is advantageous over a pure hydrocarbon system where radiative capture would occur after much longer periods of time and would lead to the release of a single 2.2 MeV $\gamma$-ray. Thus, with the gadolinium-loaded components located within the plastic scintillators, there is a very high probability of recording at least one significant energy deposition. Such a design exposes the scintillator to an increased rate of neutron background from the environment relative to ZEPLIN-III, thereby reducing diagnostic capability, but greatly increases the efficiency as an anti-coincidence detector for WIMP-like neutron recoils in ZEPLIN-III - the primary function of the device. In the default mode of operation, data acquisition for the veto will be triggered predominantly by energy deposition in ZEPLIN-III, with event-by-event veto waveforms recording for $20 \mu$ s before and $300 \mu$ s afterwards, allowing for offline searching of coincidences. Similarly, $\gamma$-ray emission, directly from components within ZEPLIN-III or otherwise, can lead to energy deposition in the scintillator. This allows coincident background events within ZEPLIN-III, predominantly from Comp-
TABLE I: Key parameters of the ZEPLIN-III veto.

\begin{tabular}{|c|c|}
\hline Total mass & $2323 \mathrm{~kg}$ \\
\hline Plastic scintillator & UPS $923 \mathrm{~A}$ \\
\hline Mass of plastic scintillator & $1057 \mathrm{~kg}$ \\
\hline Number of bars & 52 \\
\hline Typical Bulk Attenuation Length & $160 \mathrm{~cm}$ \\
\hline Mass of Gd loaded polypropylene & $756 \mathrm{~kg}$ \\
\hline Slit pitch and width & $10 \mathrm{~mm}, 2 \mathrm{~mm}$ \\
\hline Epoxy type & Rutherford Mix 71 \\
\hline Volume of epoxy & $0.125 \mathrm{~m}^{3}$ \\
\hline $\mathrm{Gd}_{2} \mathrm{O}_{3}$ loading of epoxy & $>2.7 \%(\mathrm{w} / \mathrm{w})$ \\
\hline Average Gd loading of polypropylene & $>0.5 \%(\mathrm{w} / \mathrm{w})$ \\
\hline Mass of polypropylene base & $510 \mathrm{~kg}$ \\
\hline PMT type & ETEL 9302KB \\
\hline Number of PMTs & 52 \\
\hline Typical PMT QE at $420 \mathrm{~nm}$ & $27 \%$ \\
\hline Data Acquisition & CAEN-1724 ADCs \\
\hline Waveform digitisation & $100 \mathrm{~ns}$ \\
\hline Waveform duration & -20 to $+300 \mu \mathrm{s}$ \\
\hline Contribution to ZEPLIN-III & $\begin{array}{l}<0.01 \text { neutrons } \mathrm{yr}^{-1} \\
<1000 \gamma \text {-rays } \mathrm{yr}^{-1}\end{array}$ \\
\hline Neutron tagging efficiency & $>65 \%$ \\
\hline$\gamma$-ray tagging efficiency & $>15 \%$ \\
\hline Mean neutron capture time & $35 \mu \mathrm{s}$ \\
\hline Effective threshold & $<135 \mathrm{keV}$ \\
\hline
\end{tabular}

ton scattering, to be identified. In addition to operating as an active veto, this device will provide significant diagnostic capability for ZEPLIN-III, using independent triggering and providing a measurement of cosmic-ray muon fluxes, enhanced calibration capabilities and, by being a large volume low threshold device, permitting independent measurements of the neutron and $\gamma$-ray background environment in the laboratory around ZEPLIN-III.

The veto design has several further advantages. Unlike with a liquid scintillator, where there is a possibility of leaks developing, the plastic veto scintillator bars are less of a chemical and fire hazard. Additionally, the modular design structure means that individual modules can easily be removed and repaired or modified if required without disturbing the remaining detectors or systems. As a diagnostic device, since signals generated in the veto are highly unlikely to appear in more than a few modules, a degree of directional reconstruction is possible, especially for cosmic-rays. Thirdly, with the scintillator on the outside of the polypropylene, the veto has the ability to run in several modes simultaneously, with, for example, continuous recording of background as well as timelines where ZEPLIN-III has triggered, regardless of whether the veto has detected an event. Finally, the response of the entire system can be modelled accurately by incorporating the measured performance of each unit into a simulation, as opposed to the use of global param- 
eters applied across a large volume and mass.

\section{RADIOLOGICAL CONTENT OF COMPONENTS}

At a vertical depth of $1070 \mathrm{~m}$ (2850 m water-equivalent shielding), the cosmic ray muon flux is reduced by a factor of $\sim 10^{6}$ to a level of $(3.79 \pm 0.15) \times 10^{-8}$ muons $\mathrm{cm}^{-2}$ $\mathrm{s}^{-1}$ [14]. The resultant neutrons from cosmic ray muon spallation and secondary cascades lead to a neutron scattering rate in ZEPLIN-III of $<1$ event/year for a nuclear recoil energy above $10 \mathrm{keV}$ within the central $8 \mathrm{~kg}$ of xenon [15, 16]. Thus, the most important background that must be considered comes from the local environment of the laboratory and the instruments themselves. Consequently, all components proposed for use in the veto have been assayed for their radiological content. The main contributions come from radioisotopes along the $\mathrm{U}$ and Th decay chains, which produce background neutrons via $(\alpha, n)$ reactions on other materials and from spontaneous fission, as well as numerous $\gamma$-rays. The $\beta$ decay of ${ }^{40} \mathrm{~K}$, which contaminates natural potassium at 100 ppm concentrations, generates $1.461 \mathrm{MeV} \gamma$-rays. To assess the overall neutron and $\gamma$-ray environment that the instruments will be exposed to, additional measurements of many other components have also been considered, for example the ZEPLIN-III instrument PMTs and the cavern rock. Two principal methods have been employed to determine the radioactivity content of components.

\section{A. Direct $\gamma$-ray measurements}

Direct measurements of the $\gamma$-ray emission from candidate components has been made using a high purity germanium (HPGe) detector located in a dedicated low background counting facility at the Boulby mine. The HPGe detector head was encased in an inner copper and outer lead castle leaving a $(30 \mathrm{~cm})^{3}$ test cell. The ambient $\gamma$-ray flux experienced by the HPGe detector in the absence of a test sample was then assessed through a week-long background run. A sample of material was placed close to the head and further data accrued over a period of several days. An example for a backgroundsubtracted sample spectrum is shown in Figure 3, revealing photopeaks at energies corresponding to known $\gamma$-ray emissions from $\mathrm{U}$ and $\mathrm{Th}$ chains and from $\mathrm{K}$ as well as other dominant lines of interest. To assess the quantity of an isotope that must have been present in order to generate the measured excess in the backgroundsubtracted spectrum, secular equilibrium was assumed and a GEANT4 [17] Monte Carlo simulation of the low background counting setup performed. Each simulation was individually tailored to the geometry of the sample being tested. Sensitivities at the level of $0.1 \mathrm{ppb}$ for $\mathrm{U}$ and Th, and $0.1 \mathrm{ppm}$ for $\mathrm{K}$ were achieved using this technique. The conversion between $\gamma$-rays emitted from an
TABLE II: Radiological content with statistical uncertainties where appropriate of veto components as assayed either by direct observation of $\gamma$-ray emission (HPGe) or through mass spectroscopy techniques (ICP-MS/OES). See text for further details.

\begin{tabular}{|c|c|c|c|c|}
\hline \multirow[t]{2}{*}{ Component } & \multirow[t]{2}{*}{ Mass, kg } & \multicolumn{3}{|c|}{ Radiological content } \\
\hline & & $\mathrm{U}(\mathrm{ppb})$ & $\mathrm{ppb)}$ & $\mathrm{K}(\mathrm{ppm})$ \\
\hline \multicolumn{5}{|l|}{ HPGe measurements } \\
\hline Plastic scintillator & 1057.0 & $0.2 \pm 0.3$ & $0.1 \pm 0.7$ & $0.2 \pm 0.6$ \\
\hline PTFE inner wrap & 8.9 & $1.3 \pm 0.2$ & $0.2 \pm 0.5$ & $1.2 \pm 0.4$ \\
\hline Silicone & 0.1 & $2.9 \pm 0.4$ & $0.5 \pm 0.8$ & $5.7 \pm 1.1$ \\
\hline PTFE tape & 3.1 & $3.2 \pm 1.3$ & $6.1 \pm 1.1$ & $3.9 \pm 1.0$ \\
\hline Veto PMTs & 6.2 & $38.0 \pm 0.8$ & $21.1 \pm 1.2$ & $65.5 \pm 2.4$ \\
\hline PMT preamplifiers & 0.7 & $8.4 \pm 1.7$ & $13.2 \pm 2.2$ & $10.1 \pm 1.7$ \\
\hline PMT base & 5.5 & $12.7 \pm 1.4$ & $14.8 \pm 2.4$ & $20.2 \pm 2.4$ \\
\hline Epoxy & 70.0 & $2.5 \pm 0.6$ & $0.9 \pm 0.3$ & $0.6 \pm 0.1$ \\
\hline Gd oxide & 8.0 & $0.9 \pm 0.1$ & $1.2 \pm 0.3$ & $1.7 \pm 1.1$ \\
\hline \multicolumn{5}{|l|}{$I C P-M S / O E S$} \\
\hline Copper tape & 26.0 & $1.9 \pm 0.2$ & $2.9 \pm 0.4$ & $14.0 \pm 2.0$ \\
\hline PTFE inner wrap & 8.8 & $2.0 \pm 1.0$ & $5.0 \pm 1.0$ & $<4$ \\
\hline Veto PMTs & 6.2 & $30.2 \pm 2.2$ & $30.0 \pm 3.7$ & $60 \pm 2.2$ \\
\hline PMT preamplifiers & 0.7 & $10.3 \pm 0.5$ & $29.7 \pm 3.2$ & $24 \pm 3.7$ \\
\hline PMT base & 5.5 & $13 \pm 3.4$ & $19 \pm 2.0$ & $21 \pm 3.0$ \\
\hline Polypropylene & 510 & $<1$ & $<1$ & $<5$ \\
\hline PMT mounting & 15.8 & $30 \pm 7.8$ & $<10$ & $<10$ \\
\hline Cabling & 30.2 & $110 \pm 5.4$ & $20 \pm 3.2$ & $29 \pm 7.3$ \\
\hline Connectors & 2.1 & $<10$ & $<10$ & $<4$ \\
\hline Optical gel & 0.3 & $<1$ & $<1$ & $<1$ \\
\hline Gd oxide & 8.0 & $2.5 \pm 0.5$ & $3.4 \pm 0.7$ & $<4$ \\
\hline
\end{tabular}

isotope and the source contamination level is given using the following $\gamma$-ray yields per $\mathrm{kg} \cdot$ day: $2310 \gamma$-rays $/ \mathrm{ppb}$ U, $958 \gamma$-rays/ppb Th and $285 \gamma$-rays/ppm K. A summary of the activity levels found is presented in Table II. together with the total mass of each component used in the construction.

\section{B. Mass spectrometry measurements}

To supplement some of the $\gamma$-ray measurements, and to provide a cross check of their accuracy, a subset of components was measured using different techniques at the Geosciences Advisory Unit (GAU-Radioanalytical) at the University of Southampton. Samples were dissolved and subsequently diluted in $2 \% \mathrm{HNO}_{3}$. Activity due to ${ }^{232} \mathrm{Th},{ }^{235} \mathrm{U}$ and ${ }^{238} \mathrm{U}$ was measured using an inductively-coupled plasma mass spectrometer (ICPMS), while potassium content was assessed using an inductively-coupled plasma optical emission spectrometer (ICP-OES). A calibration ${ }^{236} \mathrm{U}$ recovery tracer was added. The results from this process are also presented in Table II. Differences arise due to the assumption of secular equilibrium used to infer contamination levels in the direct $\gamma$-ray measurements, as opposed to the direct measurement of atoms using mass-spectroscopy. In all cases, upper limits from either set of measurements (HPGe or 

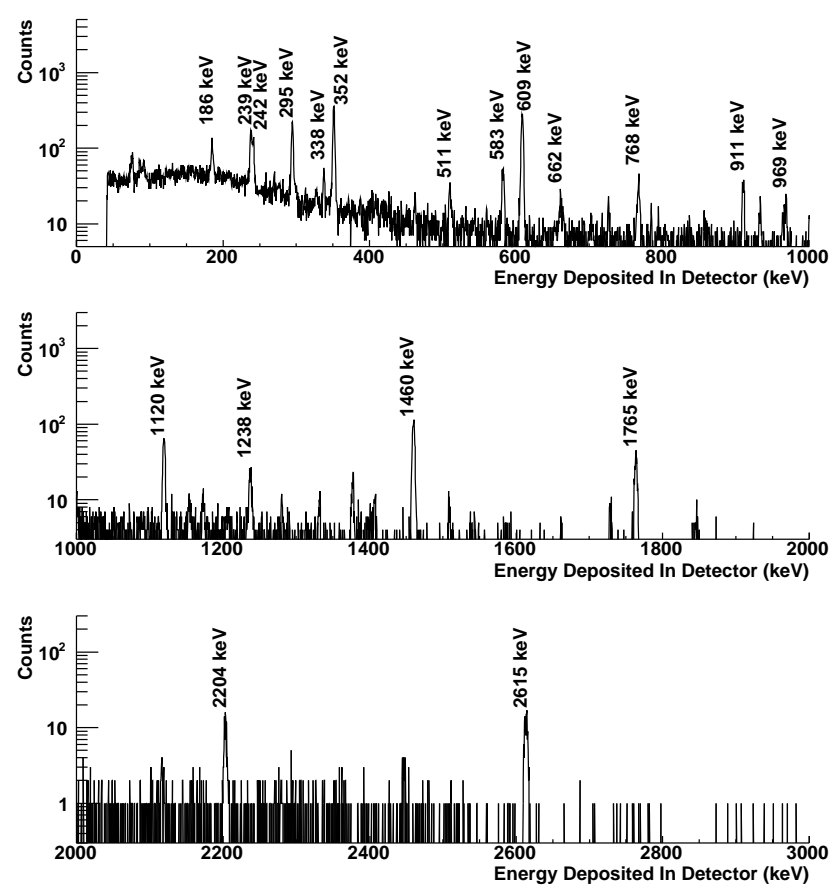

FIG. 3: Illustrative spectra from the underground HPGe detector. In this case the sample being measured was a collection of three PMTs, positioned close to the HPGe detector head. Shown is a background-subtracted exposure of 160 hours duration. The energies of $\gamma$-ray lines of interest are indicated.

ICP-MS/OES) have been used as inputs for simulations, described in Section $\mathrm{V}$. performed to assess the impact of radiological contamination in the veto components on ZEPLIN-III as well as in the veto itself.

\section{PERFORMANCE OF COMPONENTS}

The overall capability of the veto relies on the performance of individual components as well as on the geometry and methodology in which they are used. In this section we report on the technical performance of key components: the PMTs, the plastic scintillator, the front-end electronics and the data acquisition system.

\section{A. Photomultipliers}

The PMTs used are model 9302KB from ET Enterprises Ltd (ETEL). These are $78 \mathrm{~mm}$ diameter window devices with bialkali photocathode and 9 dynode stages. Their envelopes are manufactured from low background glass, with manufacturer-quoted contamination levels of $30 \mathrm{ppb}$ U, $30 \mathrm{ppb}$ Th and $60 \mathrm{ppm} \mathrm{K}$. As described in Section III A experimentally measured values using a HPGe detector in a low background environment confirm these levels.
Voltage divider networks (ETL C647BFN2-01) were fitted to low background B14A bases supplied by ETEL, with $\mathrm{U}$, Th and $\mathrm{K}$ contamination levels measured to be consistent with, or lower than, the manufacturer stated values of $13 \mathrm{ppb}, 19 \mathrm{ppb}$ and $21 \mathrm{ppm}$, respectively. To amplify the small signals from low energy events and to impedance-match the output of the PMT to the data acquisition digitisers, pre-amplifiers have been designed and manufactured that give a gain of $\sim 10$. The preamplifiers are tooled onto surface mount printed circuit boards made from light-weight low-background single-sided resin bonded paper. The PMTs and their bases (including the voltage divider network and the preamplifier board) are secured within a PVC cylinder which is itself chemically bonded directly to the scintillator. Springs mounted in the cylinder cap provide support for the PMT, pushing it into contact with the scintillator surface via BC-630 optical coupling grease. The PMTs are operated with negative bias supplied by Lecroy $1443 \mathrm{NF}$ boards within a Lecroy $1440 \mathrm{HV}$ high voltage system. All power supply and signal cabling for the photocathode-anode (k-a) HV, pre-amplifier power, and signal output, has been manufactured to order with minimal radiological contamination.

Single photoelectron (SPE) integrated pulse height spectra from dark thermionic emission have been obtained for each PMT, an example of which is shown in Figure 4. The average dark count rate is found to be approximately $300 \mathrm{~Hz}$. By design, the $10 \mathrm{MHz}$ sampling frequency of the data acquisition is sufficient for good recovery of SPE signals, allowing these to be used routinely for relative gain characterisation. These spectra were taken with no scintillator or other light sources present. For these measurements a single reference PMT was used assuring environmental and electrical uniformity across tests, with both the reference and test PMT housed in a light-tight box. The bias voltage of the test PMT was varied until the spectral peak position observed from single photoelectron emission from the photocathode was matched to that of the reference PMT. This was repeated for all PMTs.

With PMT bias voltages adjusted to yield normalised SPE spectra, the quantum efficiency (QE) and gain of each PMT was calibrated. This was performed by illuminating the test PMT photocathode with a blue LED to stimulate emission from the photocathode. The number of photoelectrons observed was then calculated using two different methods.

In the first method, a statistical approach is adopted where the number of photoelectrons detected is determined from the width of the distribution generated. The width of the peak seen from the LED is dominated by the Poissonian statistics of the photoelectron emission, i.e. $\sigma=m / \sqrt{N}$ where $\sigma$ is the measured standard deviation, $m$ is the centroid of the peak, and $N$ is the mean number of photoelectrons emitted for events in the peak. This will underestimate the total number of photoelectrons from a PMT, since it does not account for addi- 


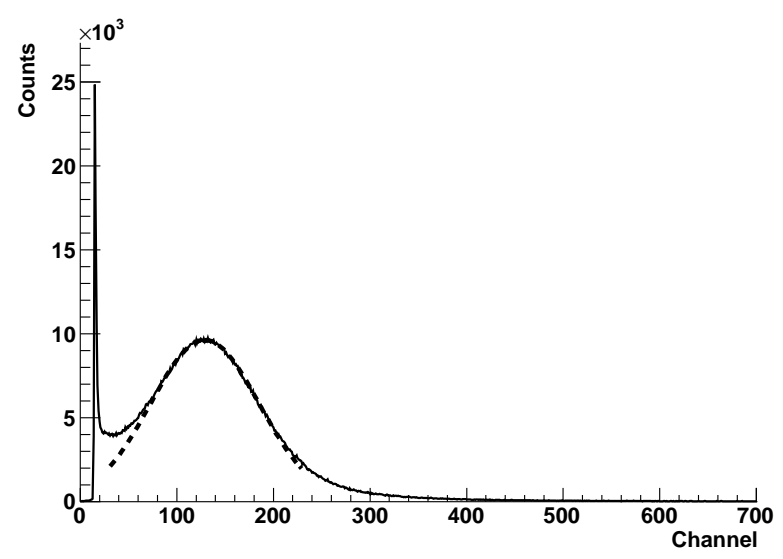

FIG. 4: A typical single photoelectron spectrum (solid line), with a partial Gaussian fit to the peak (dashed line), obtained from a PMT in the absence of a light source and with no plastic scintillator attached.

tional contributions to $\sigma$, the most important being the width of the SPE spectrum itself. However, as was the case here, this can be mitigated somewhat by generating a large number of photoelectrons such that the width of the distribution is much greater than the width of the SPE spectrum.

The second and more traditional method is to calculate the number of detected photoelectrons by simply dividing the peak position of the LED spectrum by that obtained from the single photoelectron response from dark spontaneous emission. The measured response was then normalised to absolute QE when 3 out of the 52 PMTs were absolutely calibrated at ETEL. The distribution of QEs, as plotted in Figure 5, shows good agreement between both methods used for estimating the number of photoelectrons. The gain normalised measurements confirmed the relative QEs between PMTs for sensitivities of $50 \mathrm{~A} / \mathrm{lm}$ and $200 \mathrm{~A} / \mathrm{lm}$ as quoted by the manufacturer. The mean QE at $360 \mathrm{~nm}$ is $30.1 \%$ and at $420 \mathrm{~nm}$ is $27.1 \%$.

\section{B. Plastic Scintillator: UPS-923A}

The $1057 \mathrm{~kg}$ of scintillator material is polystyrene based UPS-923A (p-terphenyl 2\%, POPOP 0.02\%) produced by Amcrys-H, Kharkov, Ukraine [18]. This material emits scintillation light with a peak intensity at $420 \mathrm{~nm}$ and with a rise time of $0.9 \mathrm{~ns}$ and decay time of $3.3 \mathrm{~ns}$. The density of UPS-923A is $1.06 \mathrm{~g} \mathrm{~cm}^{-3}$ and its refractive index is 1.52. The nominal light output for the material is stated as $55 \%$ of anthracene. The transparency of the scintillator material is determined by the so-called Bulk Attenuation Length (BAL) which is the length that reduces the initial light intensity by factor e according to the Buger-Lambert law, and is expected to be greater than $1 \mathrm{~m}$ (see later for measured values). A previous

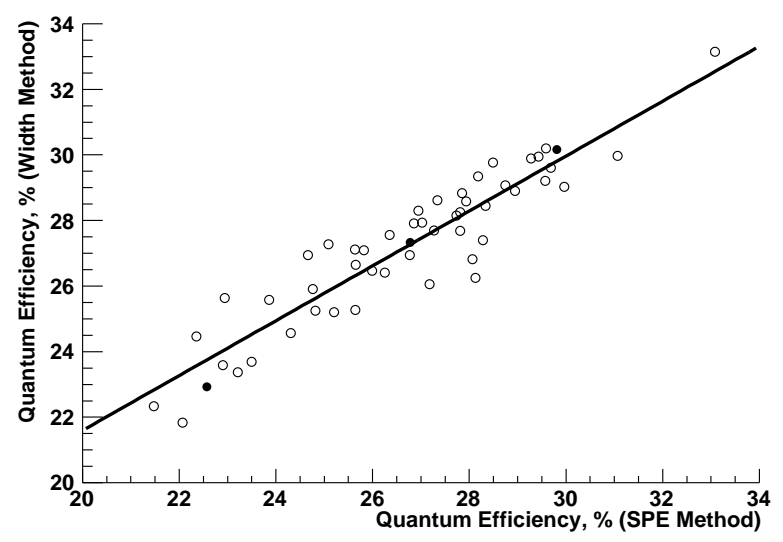

FIG. 5: The quantum efficiencies of the PMTs used in the ZEPLIN-III veto. Two methods have been used giving broadly consistent results. Three PMTs spanning the range of performance were assessed for absolute quantum efficiency externally; these are indicated by the filled symbols. A linear fit through the data points is drawn to highlight the bias between the two methods used.

study by the manufacturers suggests this material to have long-term stability, such that there is no natural degradation reported over a 12 year period [19].

To minimise loss of light within a scintillator bar each piece has been wrapped along its length with PTFE sheet giving it a high diffuse reflectivity. Experimental tests were performed comparing a number of diffuse and specular reflective wrapping materials including white paper, aluminised Mylar, Vituki 3M [20] and other PTFE-based sheeting. Three layers of $76 \mu \mathrm{m}$ thick PTFE film [21] was found to provide the most cost effective option, increasing light collection by up to a factor of 2 at the PMT. At the far end from the PMT a specular reflective aluminised Mylar foil is placed that acts as a mirror to improve the uniformity and increase the effective BAL of the unit. The PMT is optically coupled to the scintillator with BC-630 silicone-based optical couplant and is housed in a PVC cylindrical tube chemically bonded to the scintillator. The entire assembly has then been wrapped in black opaque PVC sheet 22] to provide light tightness. Figures 6 and 7 show unwrapped and wrapped plastic scintillator barrel bars.

The performance of each of the 52 pieces has been assessed individually as follows. For each fully wrapped plastic scintillator module, spectra of the PMT response to a ${ }^{22} \mathrm{Na}$ calibration source were recorded for each of six positions along the length. To ensure consistency, the same PMT, voltage divider network, preamplifier and operating bias were used in these measurements. Additionally, the use of optical grease was omitted to reproduce conditions as exactly as possible across scintillators. It was found that without the optical grease the number of photoelectrons detected is approximately halved, and this is confirmed by Monte Carlo simulations [23]. 


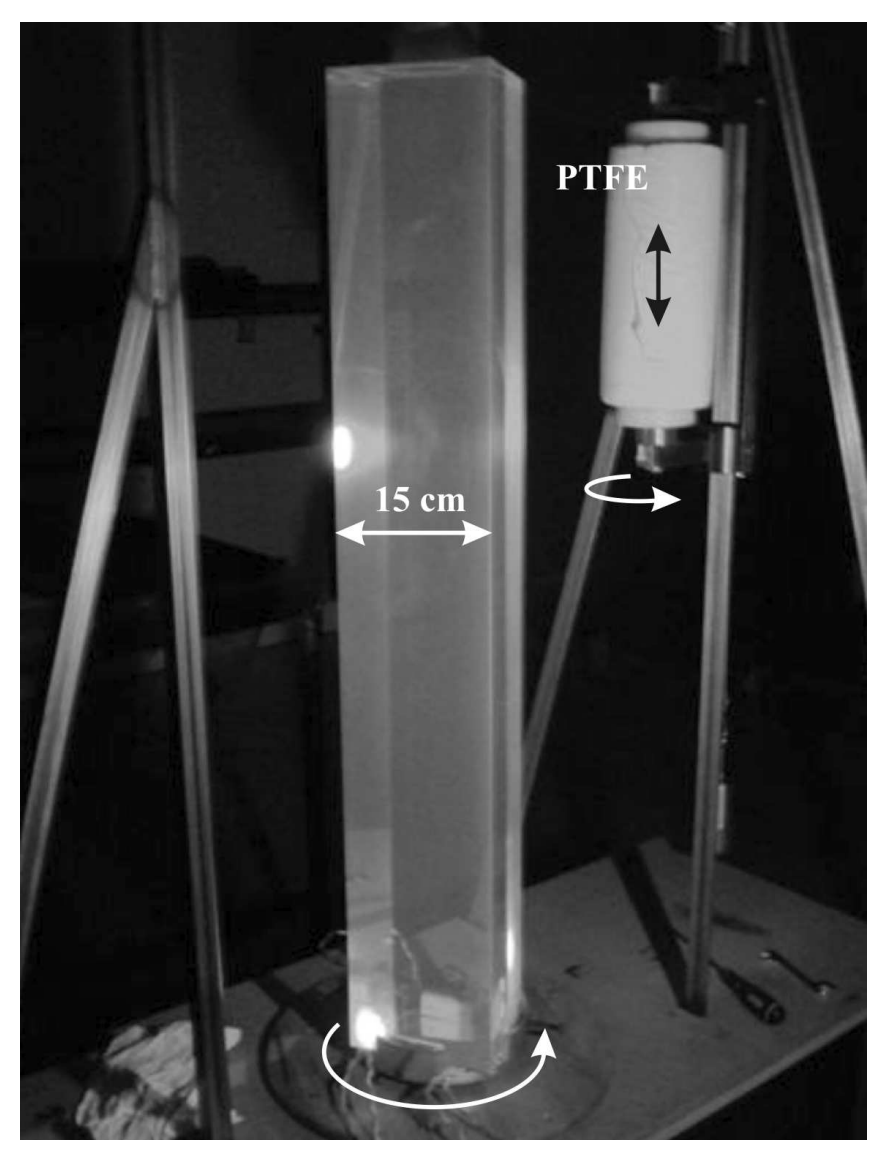

FIG. 6: Photograph of an unwrapped barrel bar of plastic scintillator with the apparatus used to wrap bars with PTFE to increase light collection.

The measurements were performed in a surface laboratory of predominantly concrete construction, and thus, for plastic scintillators of this size, a high rate of $\gamma$-rays from the local environment was observed. To reduce this contribution, events seen in the plastic scintillators were required to be in coincidence with events in a 3 " diameter, 3" long NaI scintillation detector placed on the other side of the radioactive source from the plastic scintillator. Since ${ }^{22} \mathrm{Na}$ decays by $\beta^{+}$emission this greatly enhanced the fraction of events caused by the $511 \mathrm{keV}$ annihilation and $1275 \mathrm{keV}$ de-excitation $\gamma$-rays. However, since $\gamma$-rays produce largely Compton electrons in organic scintillators at these energies, the trigger signal of the $\mathrm{NaI}$ detector can come either from another $\gamma$-ray, or from the Compton-scattered $\gamma$-rays detected in the plastic scintillator. In the latter, the shape of the energy spectrum may be effected since Compton electrons in the scintillator deposit lower energy and yet the scattered $\gamma$-ray retains sufficient energy to trigger the NaI. Monte Carlo simulations of the experimental setup confirmed that, with the geometry used for these measurements, the rate of such events relative to true coincidence triggers from separate gamma-rays was negligible.

A typical Compton spectrum is shown in Fig. 8, reveal-

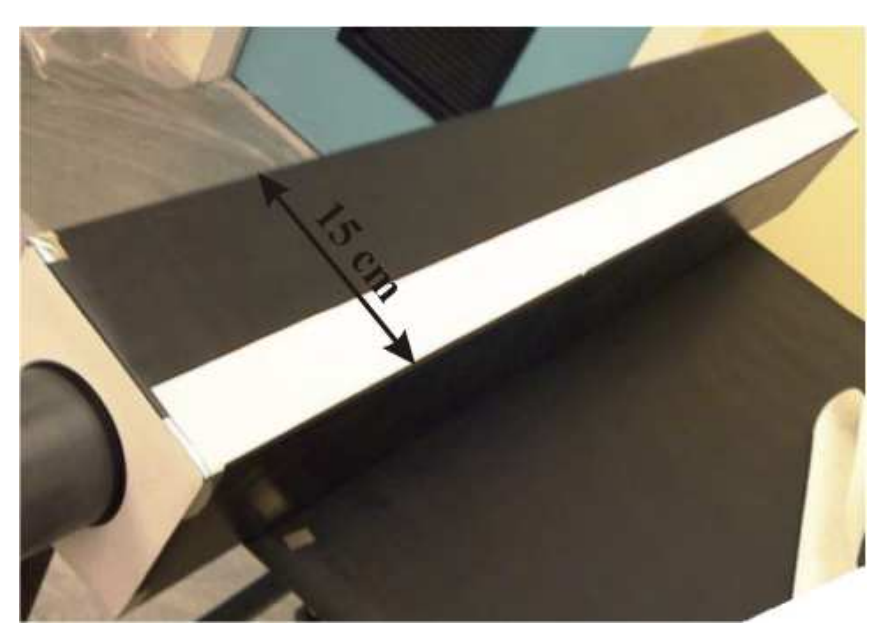

FIG. 7: Photograph of a wrapped plastic scintillator module. Around the bare plastic is a layer of PTFE, a mirror at the opposite end from the PMT, and black wrapping to light tighten the module. The PMT itself is housed in a light tight cup bonded to the scintillator.

ing features corresponding to the $511 \mathrm{keV} \gamma$-rays, as well as a weaker peak corresponding to the $1275 \mathrm{keV} \gamma$-ray emission following $\beta^{+}$decay of ${ }^{22} \mathrm{Na}$, and a background well described by an exponential trend. The calibration between observed Compton peak position and number of photoelectrons has been used to allow this spectrum to be plotted in terms of numbers of photoelectrons. A two-partial-Gaussian-plus-exponential fit was made to the data and the movement of the centroid of the partial Gaussians and the Compton edge as a function of the position of the source along the length was used as the indicator of the technical attenuation length (TAL). The BAL describes the attenuation of photons in a beam due to the scintillator only, whereas the TAL parameter is an auxiliary parameter. The difference between the and BAL comes from the fact that the real light path from the vertex of the interaction to the PMT is longer than a straight line path, since most of of the light reaching the PMT has been reflected at the surface of the scintillator bar through total internal reflection. The measured TAL combines geometry effects (light path, reflection), light attenuation, wavelength shift (due to wavelength dependent absorption and re-emission) and wavelength dependent quantum efficiency of the photo cathode. In the case of the modules measured here, a highly-reflective specular mirror surface has also been placed at the opposite end to the PMT. Consequently, one expects, to first order, the response as a function of distance from the PMT to vary as the sum of two exponentials, one describing the reduction due to the separation of PMT and source position, and one which describes the reduction due to the distance between the PMT and the image of the source in the mirror:

$$
S(x)=A e^{-x / T}+A e^{-(2 l-x) / T}
$$

where $S(x)$ is the centroid of the measured photopeak, 
$x$ is the distance from the PMT face, $l$ is the length of the scintillator module (the distance between the PMT and the mirror), $T$ is the TAL and $A$ an arbitrary scaling constant. In principle, one could use a different scaling for the term describing the response due to reflections from the mirror, effectively accounting for imperfect reflection from it, but the quality of the data available did not justify an additional free parameter. The measurements of TAL have been complemented with cosmic ray muon measurements in which the $\mathrm{NaI}$ coincidence detector was replaced by two small plastic scintillator detectors placed above and below the test piece. In this case the spectra obtained are well described and fitted by a Landau distribution, and again the response as a function of measurement position determined. Consistent results were found for the TAL measured either with the ${ }^{22} \mathrm{Na}$ source or with cosmic rays.

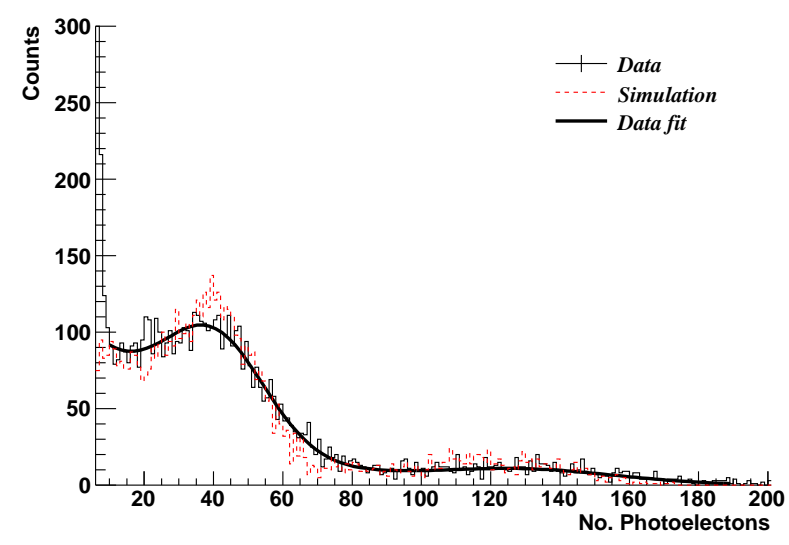

FIG. 8: Typical spectrum (solid histogram) showing the response of a single barrel module of plastic scintillator to irradiation with a ${ }^{22} \mathrm{Na} \gamma$-ray source. See text for further details of the source geometry. The solid line is the result of a fit to the data assuming partial Gaussians for the peaks attributed to the 511 and $1275 \mathrm{keV} \gamma$-rays and an exponential to describe the background. Also shown (dashed histogram) is the result of a Monte Carlo simulation of this exposure.

To assess whether the measured TALs are consistent with the expected BAL of the material, GEANT4 Monte Carlo simulations of these tests have been performed (see Section $\nabla 7$. These included the geometry of the plastic scintillator, laboratory and source, as well as physical parameters such as the light output of the plastic, gain and quantum efficiency of the PMT (see section IVA). The simulations include full tracking of scintillation photons from $\gamma$-ray energy deposition through to incidence on PMT photocathode and stochastic generation of photoelectrons, allowing realistic spectra to be generated. The lighter histogram in Figure 8 shows the result of the simulation tailored to that particular measurement's geometry, illustrating that the key features are very well reproduced. The simulations are repeated for different values of BAL, each time generating spectra for differ- ent source target positions, and these spectra evaluated in the same manner as was done for real data to extract a TAL. Figure 9 shows the position of the centroid of the peak attributed to the $511 \mathrm{keV} \gamma$-ray as a function of the source location both for real data from one of the barrel modules, and evaluated from Monte Carlo simulations of those exposures with an appropriately chosen value of BAL. This BAL is then taken as corresponding to the experimental value of TAL indicted by the data. Lines have been added to guide the eye in this figure and in subsequent figures where appropriate. As expected, for each scintillator bar geometry a monotonic dependence between BAL and TAL is found, with TALs having smaller values than BALs, and the most uniform geometry bars having the highest TALs. These results are summarised in Figure 10. We find a large dispersion in BAL, ranging from $\sim 60 \mathrm{~cm}$ to greater than $2.5 \mathrm{~m}$, with significant variations even between bars polymerised in the same batch. The measurements allow the matching of individual bars of plastic scintillator to particular PMTs, matching lower BAL with higher QE to provide a more uniform performance across the entire veto and informing the placement of scintillator modules within the array. This also provides input for the Monte Carlo simulations described in section $\overline{\nabla B}$ in which the overall performance of the whole veto system is evaluated.

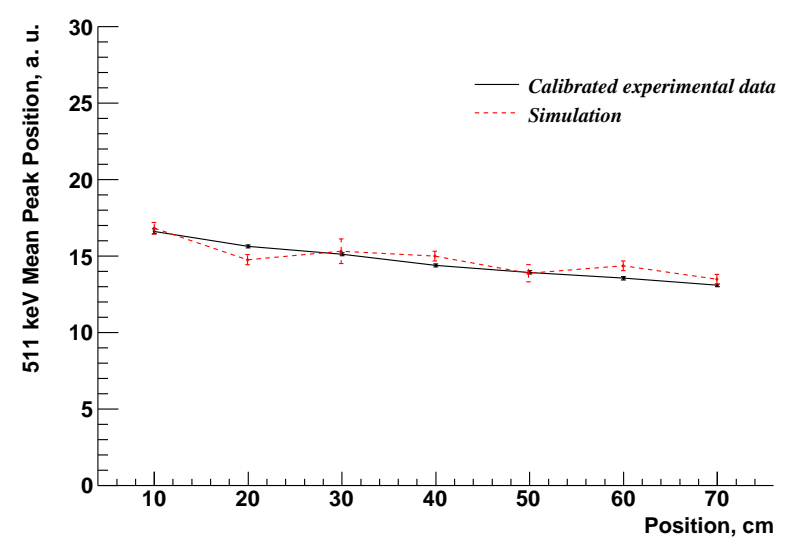

FIG. 9: Variation of the peak position from the $511 \mathrm{keV} \gamma$-ray with source location both for data from one of the $1 \mathrm{~m}$ long barrel modules and evaluated from Monte Carlo simulations of those tests. Lines have been drawn to guide the eye.

Although many processes contribute to the scintillation light emission [24, 25], the absolute light output of the plastic scintillator can be calculated by matching the measured and simulated spectra. A consistent value of $\sim 5500 \mathrm{ph} / \mathrm{MeV}$ was required for all bars, regardless of which polymerisation batch they came from. This is lower than expected, as $55 \%$ of anthracene would correspond to nearer $8500 \mathrm{ph} / \mathrm{MeV}$, necessitating the use of reflective wrapping around the scintillators.

During normal operation of the veto a blue LED unit will continuously flash 52 separate optic fibres coupled 


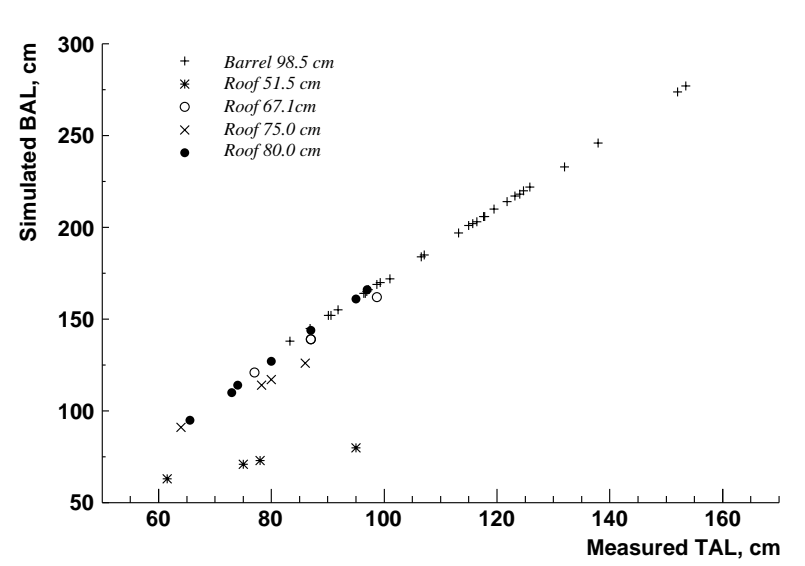

FIG. 10: Measured technical attenuation length (TAL) and Monte Carlo predicted bulk attenuation length (BAL) for each bar of plastic scintillator.

directly to the ends of the plastic scintillator modules furthest from the PMT. This will allow any degradation of the scintillator performance over time to be monitored.

\section{Data Acquisition}

The outputs from the PMT preamplifiers are fed to CAEN V1724 ADCs housed in a VME8011 crate with CAEN V2718 PCI bridge communicating via optical link to a dedicated Linux-based data acquisition computer. The digitisers have $\pm 2.25 \mathrm{~V}$ input range with 14-bit resolution and $40 \mathrm{MHz}$ bandwidth with a sampling rate of up to $100 \mathrm{MS} / \mathrm{s}$ simultaneously on each channel, with internal buffering to ensure zero deadtime performance at expected data rates. Custom made run-control software provides a graphical user interface for beginning and terminating data acquisition runs with easily changeable run parameters and toggles over various operation modes. The software also provides remote capabilities to fully automate instrument operation. A screenshot of this interface is shown in Figure 11, Amongst the most important operation modes are the veto 'slave' mode and calibration/diagnostic 'master' mode.

In slave mode, all modules take an external trigger sourced from the ZEPLIN-III instrument. As such, the veto is guaranteed to record data when ZEPLIN-III itself has recorded an event. Besides simplicity of design, this minimises data volume and obviates the need for a separate hardware-enforced trigger and associated efficiency loss at low energies. Additionally, spontaneous photoelectron emission from the PMTs, present in the timelines recorded where pulses are absent, can be used for equalising gains, calibrating and monitoring stability of the PMTs without acquiring dedicated data, where conditions between data runs may vary.

Finally, since even with high energy depositions in the veto only a few of the scintillator modules are involved, the remaining modules provide diagnostic information at the time of the event. Monte Carlo simulations indicate background $\gamma$-ray events depositing $>200 \mathrm{keV}$ distribute $>90 \%$ of the energy in 6 modules or fewer (see section $\mathrm{VB}$.

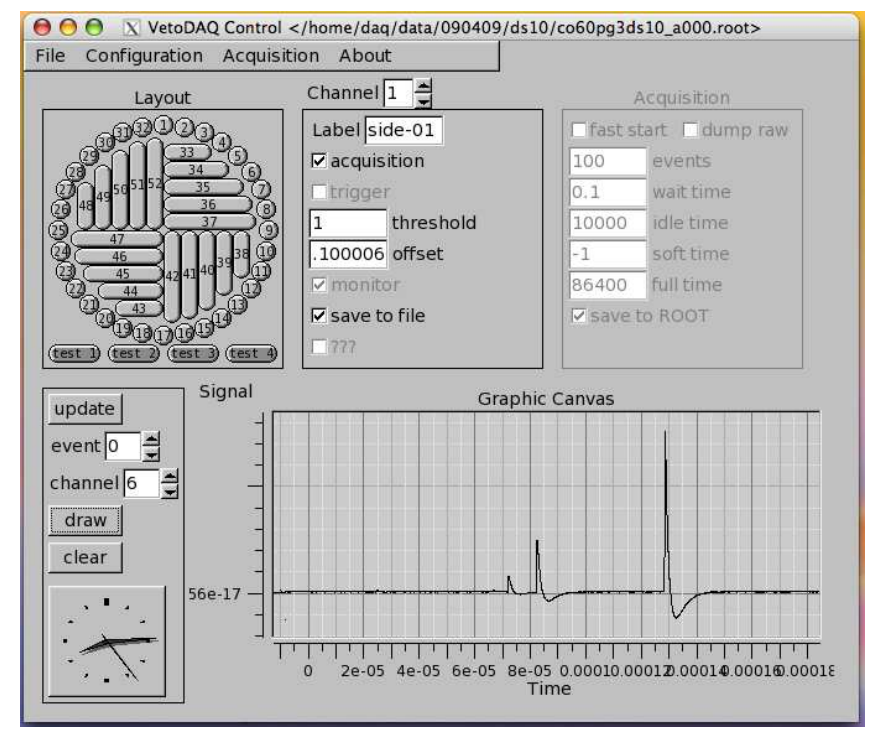

FIG. 11: Graphical user interface of veto data acquisition.

The expected time delay between signals in ZEPLINIII and in the veto defines the requirements for the duration of the waveforms to be be recorded. Coincident $\gamma$-ray events will be essentially simultaneous. For single scatter neutron recoils within ZEPLIN-III, where the veto signal is provided by energy deposition of $\gamma$-rays following neutron capture on gadolinium, the coincidence is expected to have a mean delay of $\sim 35 \mu$ s (see Section $\mathrm{V} \mathrm{Cl}$ ). However, for small energy deposits in ZEPLIN-III, the trigger is in fact provided by the $\mathrm{S} 2$ electroluminescence, which is itself delayed by up to $17 \mu \mathrm{s}$ (due to the time it takes electrons to drift through the xenon). It is therefore necessary that a period of pre-trigger data is also recorded: values of $20 \mu$ s pre-trigger and $300 \mu$ s post-trigger have been chosen.

An example of a waveform recorded in the veto data acquisition is presented in Figure 12, This event corresponded to irradiation by a ${ }^{60} \mathrm{Co}$ source located approximately half way along the length of one of the barrel modules. By design, the gain of the system is such that $\gamma$-rays of about this energy populate the mid range of the 14-bit ADC. The baseline noise is observed to be between typically $2 \mathrm{mV}$, or about $30 \mathrm{ADC}$ channels. In addition to the $\gamma$-ray event at $60 \mu \mathrm{s}$, a typical single photoelectron can be seen at $230 \mu$ s, clearly visible above the noise level.

The master diagnostic/calibration mode takes either a forced trigger to allow the veto modules to record effectively continuously without imposing a signal threshold for triggering, or can be set to record data when a defined number of scintillator modules detect a signal above 


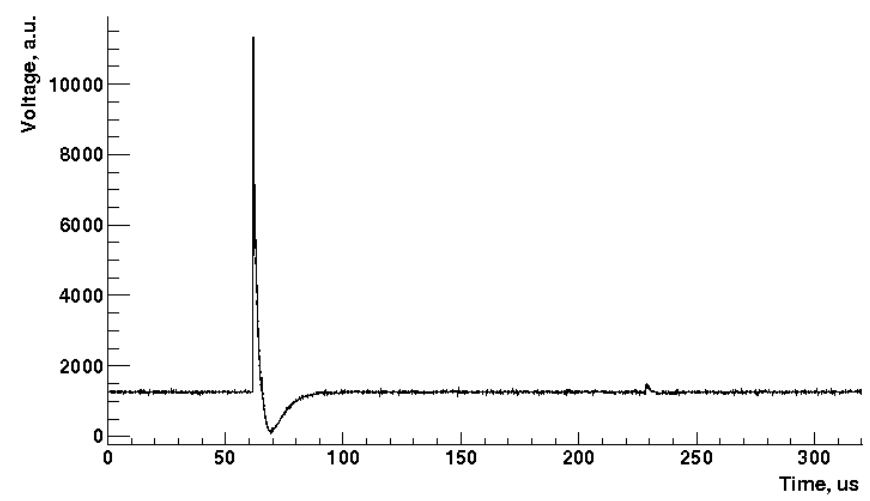

FIG. 12: An example waveform from a barrel module plastic scintillator in response to a $\gamma$-ray from a ${ }^{60} \mathrm{Co}$ source, with the pulse starting at $60 \mu \mathrm{s}$. Also clearly visible is a single photoelectron at $230 \mu \mathrm{s}$.

a defined threshold. These are necessary for in situ calibration of the veto device and for recording background data in all modules. As such, when operating in this master mode, the veto system can operate as a detector independently of ZEPLIN-III if required.

The default operating mode will be a combination of these triggering techniques, concurrently recording a level of background without hindering the coincidence detection capabilities: the veto will trigger whenever ZEPLIN-III observes a signal, regardless of the presence of a signal in the veto, but it will also trigger whenever the veto observes a signal in two or more modules above some threshold set to exclude noise and maintain a low count rate. Since the acquisition can run with zero deadtime by storing events in its internal buffer, there is no possibility of missing a ZEPLIN-III trigger. To maintain accurate synchronisation between ZEPLIN-III and the veto data acquisitions, a dedicated hardware unit produces a digital time stamp which is sent to both acquisitions so that events can be correlated with $100 \mathrm{~ns}$ accuracy.

Finally, taking advantage of the segmented nature of the veto, PMT outputs from the 20 roof modules are fed into a dedicated triggering unit that passes signals through to the ADC inputs but also sums them and shapes this summed signal. The shaping parameters are set such that if the unit finds a pulse in any module that is characteristic of a cosmic ray muon, all 52 veto channels are triggered and the event recorded, regardless of a coincident event in ZEPLIN-III. This is a valuable diagnostic allowing measurement of the cosmic ray flux through the laboratory, as well as providing a measure of muon induced neutron background in the local environment.

\section{MONTE CARLO SIMULATIONS}

Monte Carlo simulations have been used extensively in this work, both to characterise individual components, for example correlating the BAL and TAL responses of individual plastic scintillator modules, and as a tool for optimising and understanding the overall performance of the veto.

The requirements for this Monte Carlo simulation are relatively demanding. The programs should be capable of modelling the transport and interactions of particles (essentially neutrons and $\gamma$-rays) internal and external to ZEPLIN-III and the veto through to the production of electron and nuclear recoils. The program must then simulate the physical processes involved in the generation of the optical response to scintillation (and electroluminescence in ZEPLIN-III), and finally generate the response for all channels in order to produce realistic datasets. The existing ZEPLIN-III Monte Carlo simulation package [2], and the veto simulations developed here, have utilised the GEANT4 toolkit [17], which is capable of fulfilling all of these requirements.

In this section we first present the general features of the simulation, leading to calculation of the expected ability of the veto to reject both neutron and $\gamma$-ray events that, based on ZEPLIN-III data alone, might be misidentified as WIMPs. A key feature of the veto design is the inclusion of gadolinium to enhance the response to neutrons. We have used the Monte Carlo methodology to explore the dependence of the design to the amount and location of gadolinium used, and this is presented. We then bring together the measurements of radiological content and performance of all the veto components to estimate the absolute event rate that the veto will experience, and its impact on the rejection performance of the instrument. Finally, we utilise the Monte Carlo simulations to explore effects such as misalignment between modules introduced during assembly. The Monte Carlo simulation has also been used to inform numerous other choices not mentioned, for example the geometry of the active and passive modules.

\section{A. General features of the simulation}

The GEANT4-v9.1 Monte Carlo toolkit has been used. All simulations included accurate geometry of the plastic scintillators, their wrapping including the $95 \%$ specular reflective mirror at the far end, the PMTs including envelope, photocathode, internal elements and PVC support structure and optical grease (in terms of changes to refractive indices). Also incorporated are the geometry and materials of the surrounding area; for simulations of the whole veto this includes an extensive description of the Boulby laboratory with full details of ZEPLIN-III itself, the Gd and all related processes, the local polypropylene and lead shielding, the building, and rock walls. By varying the depth of rock included in the simulation it was 
found that a depth of $3 \mathrm{~m}$ for neutrons and $0.25 \mathrm{~m}$ for $\gamma$-rays is sufficient to accurately simulate the flux emanating from these surfaces $(<1 \%$ contribution to uncertainty). For simulations of measurements made in surface laboratories, the details of the walls, tables and other large objects in the vicinity were included as necessary. The light yield and BAL of the scintillator and the single photoelectron response and the QE of the PMTs are taken from measurements described earlier.

Simulations have been performed for radiation originating from the cavern walls, from ZEPLIN-III components, from the veto components and from calibration sources and, in each case, measured values of activity have been used. In the case of $\mathrm{U}$ and Th, the SOURCES4C [29] code has been used to generate the emission spectra for neutrons. All particles and their secondaries are tracked. The location, size and event time of all energy depositions are recorded.

A nuclear recoil energy deposition generates much less electronic excitation (primary scintillation and ionisation) than an electron recoil event of similar energy due to 'quenching'. A quenching coefficient is defined as the ratio of the amount of light induced by a recoil nucleus to the amount of light induced by an electron of the same kinetic energy. A (conservative) quenching coefficient of 0.1 is assumed for neutron energy depositions occurring in the plastic scintillator [26]. Since the efficiency for rejecting coincident events with ZEPLIN-III comes from detection of $\gamma$-rays, a large variation in quenching factor of the plastic scintillator makes negligible difference to the results presented.

Each energy deposition occurring within an active volume is converted to a number of photoelectrons seen by the PMT. This has been performed in two ways. Firstly, an option in the Monte Carlo simulation allows each of these energy depositions to be converted to scintillation photons, and for these scintillation photons to be tracked to the photocathode, with losses occurring at points of reflection and due to the bulk attenuation. Rayleigh scattering is included. Poisson statistics are then applied to determine how many photoelectrons are generated. Since this is effectively a further complete simulation for each energy deposition, this option is slow and has been used only where necessary, principally to correlate measured TAL values with intrinsic BAL of the scintillators, and to allow the light emission of the scintillator to be determined. The second much faster option is to simply apply the expected loss due to the measured TAL given the distance from the end of the scintillator. Since the measured and simulated TALs agree, a further refinement to the simulation has been to use the Monte Carlo to estimate the fully 3D-dependent TAL throughout the scintillator, rather than a single mean value that describes the unit as a whole.

\section{B. Overall Veto Performance}

The central function of the veto detector is to facilitate rejection of events that, on the basis of ZEPLIN-III data alone, could be mistaken for WIMPs. The principal characteristics of candidate events in ZEPLIN-III are that they have an electroluminescence-to-scintillation ratio characteristic of nuclear recoils, occur within the fiducial volume, and cause an energy deposition of $\leq 50 \mathrm{keVnr}^{1}$. The complete end-to-end simulation has been performed with all energy depositions in ZEPLIN-III and the veto recorded. While for the first science run the main contribution to background was the activity of the PMTs of the ZEPLIN-III device itself, after the upgrade it is expected that no single source will dominate overwhelmingly - the contributions from PMTs, components and from the rock walls being similar. Differences arise due to the activity levels particular to each case, and due to the propagation of the radiation from point of origin through to detection. Thus, the efficiency of the veto in rejecting background neutrons has been estimated by considering each of these contributions separately, and then adding the contributions appropriately.

In its simplest mode, an event that satisfies the ZEPLIN-III criteria for a WIMP can be rejected by the veto if any one of the scintillator modules registers, within an appropriate time window $(-20$ to $+300 \mu \mathrm{s})$, a signal significantly above background. These are dubbed 'tagged' events, and a tagging efficiency can be calculated as a function of the veto signal size (see Figure 13). A reasonably weak dependence on veto threshold is seen, with a maximum tagging efficiency at zero threshold of $80 \%$ possible: about $20 \%$ of neutron background events that satisfy the WIMP-search criteria in ZEPLIN-III have no interaction in any of the veto modules. In reality, a threshold of 6 photoelectrons in any single scintilator is sufficient to provide a minimal accidental coincidence contribution to overall event rate, and therefore this has been used as the conservative hard threshold within any module in simulations of the veto. Lower limit efficiencies derived from this threshold determine the minimum neutron and gamma-ray background reduction in ZEPLINIII. For the barrel modules, 6 photoelectrons corresponds to a typical energy deposition of $135 \mathrm{keV}$ at the far end and it is predicted that $>65 \%$ of background neutrons that could otherwise have been misidentified as WIMPs will be rejected by the veto with this threshold. However, as stated, since there is no hard threshold trigger for the veto when triggering in its slave mode, a higher

[1]For a given event in the liquid xenon the (quenched) nuclear recoil energy may be determined from the scintillation signal, however, it is more convenient to calibrate the detector using electron recoils. This leads to the use of two energy scales: keVee (keV electron equivalent) and $\mathrm{keVnr}$ (keV nuclear recoil). The tradition in the field is to use $122 \mathrm{keV} \gamma$-rays from a ${ }^{57} \mathrm{Co}$ source to set the energy calibration. 
tagging efficiency for coincident events (up to a maximum of $80 \%$ ) is achievable. This represents a substantial improvement on previous anti-coincidence systems such as the veto used by the ZEPLIN-II experiment [27, 28], which had a neutron tagging efficiency of approximately $50 \%$, with a similar $\gamma$-ray tagging efficiency.

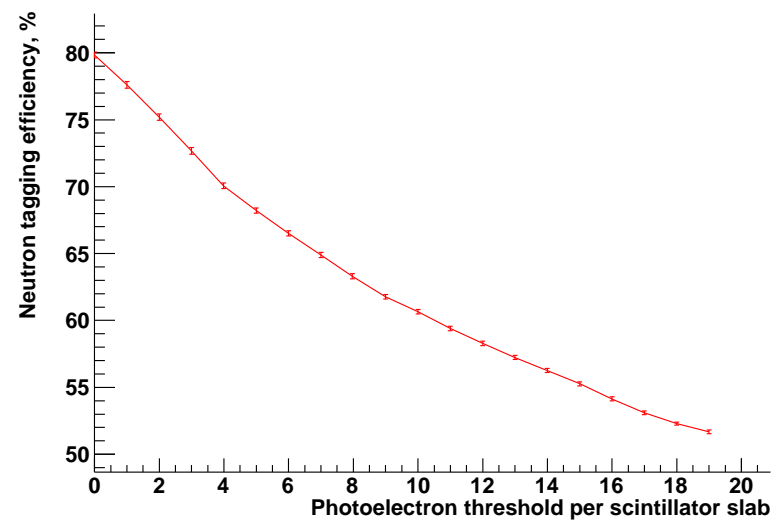

FIG. 13: Tagging efficiency of the veto for neutron events in ZEPLIN-III as a function of the signal size seen in at least one module of the veto, in terms of photoelectrons.

Figure 13 shows the neutron background rejection power of the veto based on signals above threshold in single scintillator modules only. In principle, it could be possible to increase the tagging efficiency further by searching for smaller energy depositions occurring in multiple modules simultaneously. Figure 14 extends Figure 13 to explore the summed energy deposition in multiple scintillator modules. Since there exists no energy threshold for coincidence veto events when in slave mode of operation it is possible to apply not just single module thresholds, but a threshold on the sum energy of a veto event. In this case a veto event would be defined as the sum of all veto modules with signals above a minimal threshold as low as 1 photoelectron and occuring in coincidence with one another within a short time window of a few $\mu$ s duration. The former condition takes advantage of the mutliple $\gamma$ rays emitted following Gd capture causing interactions in multiple modules to maximise the tagging efficiency of the veto system. The latter condition restricts the number of random coincidence events with ZEPLIN-III by demanding all $\gamma$-ray signals seen in multiple modules belong to the same event constrained within the timing window. Since $320 \mu$ s timelines are recorded for each module a number of such events may be observed. Figure 14 shows the neutron tagging efficiency of the veto as a function of the sum of photoelectrons detected from each modules registering at least one photoelectron. It is seen that the tagging efficiency has a much stronger dependence on the number of scintillator modules than on the signal size. With a summed signal of 6 photoelectrons the nominal $65 \%$ tagging efficiency is acieved from any single module registering 6 or more photoelectrons.
However, this efficiency is supplemented by events where the 6 photoelectrons are distributed, in any hit pattern, across multiple modules and the efficiency is increased to almost $70 \%$. The scope for improvement is limited to a maximum of approximately $80 \%$ since, as seen in Figure 13, some $20 \%$ of candidate events result in no energy deposition in the veto at all.

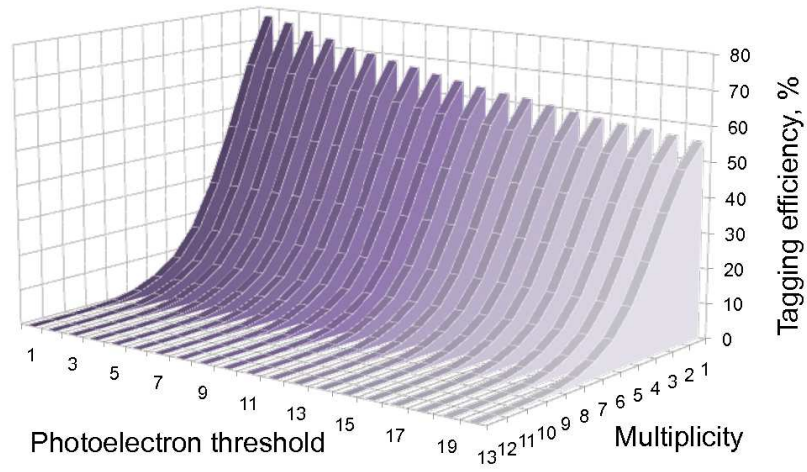

FIG. 14: The efficiency for the veto to tag background neutron events that, based on ZEPLIN-III data alone, could have been misidentified as WIMPs, as a function of the multiplivity of the event and the summed number of photoelectrons across all modules of the veto. The multiplicity is defined as the number of modules contributing to the summed signal with at least one photoelectron.

ZEPLIN-III has very good ability to discriminate between electron recoils, principally from $\gamma$-rays, and nuclear recoils from neutrons or candidate WIMPs. However, with sufficient exposure some $\gamma$-rays can still leak into the nuclear recoil acceptance region. Some fraction of these are likely to have also deposited energy in the veto, and thus could be rejected. Simulations have been performed in which $\gamma$-rays were emitted from the known activities of the rock walls, laboratory, shielding, and from the detector components. Instances in which energy was deposited in both ZEPLIN-III (2-16 keVee, single scatter in the fiducial volume) and in one or more of the veto plastic scintillators were searched for. The resulting efficiency for the veto to reject $\gamma$-rays that otherwise might have been misidentified as WIMPs, has been evaluated as a function of the number of photoelectrons seen in at least one module of the veto, and is presented in Figure 15. It is predicted that with a threshold of 6 photoelectrons being seen in a single module, $\sim 15 \%$ of background $\gamma$-rays depositing $2-16 \mathrm{keVee}$ through single scatters in the ZEPLIN-III fiducial volume can be rejected by the veto.

\section{Optimisation of gadolinium loading}

The adopted design relies heavily on the inclusion of gadolinium loaded into the polypropylene pieces of the veto. The very high neutron capture cross section of 


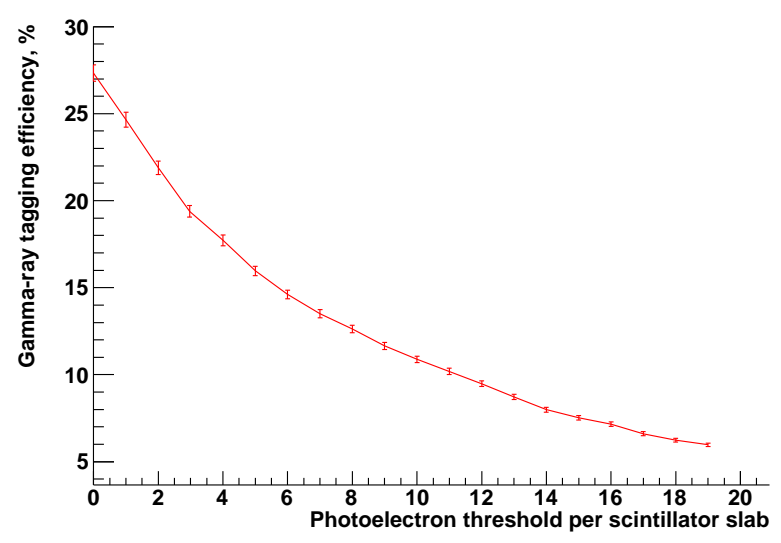

FIG. 15: $\gamma$-ray tagging efficiency of the veto as a function of the number of photoelectrons seen in at least one plastic scintillator.

${ }^{157} \mathrm{Gd}$, combined with the subsequent emission of up to $8 \mathrm{MeV}$ of $\gamma$-rays, is the principle reason for the high neutron tagging efficiency. Removing the Gd entirely results in the neutron tagging efficiency for this geometry to drop to below $45 \%$ for a 6 phe threshold. The results presented so far are for a loading of $0.5 \%$ by weight, which was the value eventually adopted; here we explore the effect of different concentrations and distributions of gadolinium.

The incorporation of $\mathrm{Gd}$ into the polypropylene has two main effects. Firstly, increasing the fraction of gadolinium increases the fraction of internal neutrons that are captured by the gadolinium isotopes, as opposed (mainly) to capture on the hydrogen content of the polypropylene and plastic scintillator. Since the ${ }^{157} \mathrm{Gd}$ capture in particular is followed by emission of several energetic $\gamma$-rays, this also tends to increase the tagging efficiency. This is illustrated in the upper panel of Figure 16 in which an exposure to neutrons from known background sources of the entire ZEPLIN-III - veto system has been simulated. For events that satisfy the WIMP search criteria (fiducial volume, $\leq 50 \mathrm{keVnr}$ ) the fraction that result in 6 or more photoelectrons in a single module of the veto is shown, as a function of the gadolinium loading. A gradual increase in tagging efficiency is seen up to about $0.5 \%$ fraction by weight. Above this little further gain is made, indicating that at this level of loading, essentially all neutrons that thermalise in the polypropylene are captured by the gadolinium.

The second effect of including gadolinium is that the mean time before neutron capture is reduced. In terms of experimental infrastructure, a short capture time is desirable because it allows a larger fraction of coincidences to be identified for a given coincidence time window, or alternatively, for a shorter window to be used reducing data volume and the false-coincidence rate. The mean time between tagged signals in ZEPLIN-III and the veto, as a function of gadolinium concentration, is shown in the lower panel of Figure 16. Here we see that above about a
$1 \%$ loading, further increase ceases to reduce the capture time, indicating that capture is occurring very soon after thermalisation.

Simulations have also shown that neutrons are effectively moderated to thermal energies whether they originate externally to the veto (such as from the rock around the laboratory) or from within the internal volumes enclosed by the veto (such as from ZEPLIN-III itself). External neutrons from the rock predominantly undergo radiative capture on $\mathrm{H}$ within the scintillator since very few are able to penetrate the $15 \mathrm{~cm}$ into the Gd-loaded polypropylene. The mean neutron energy at capture is $0.035 \mathrm{eV}$. On the other hand, internal neutrons are captured on $\mathrm{Gd}$ and, at $0.5 \% \mathrm{Gd}$ concentration, the mean energy before capture is $0.045 \mathrm{eV}$ (a slightly higher value due to the enhanced capture cross section relative to $H$ ). In both cases, neutrons entering the veto are effectively moderated and captured within the veto acquisition time window of $300 \mu \mathrm{s}$, with a mean time of $35 \mu$ s for the internal neutrons.

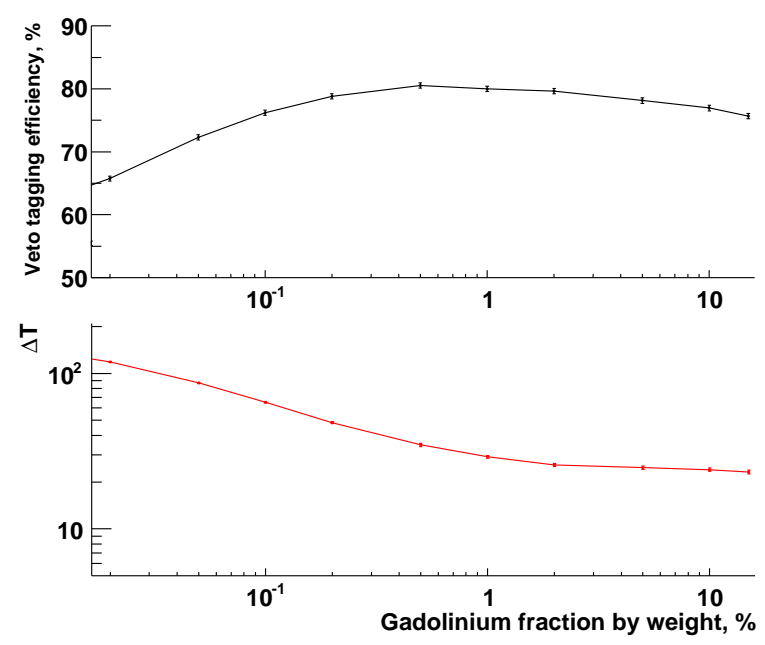

FIG. 16: Monte Carlo simulations of the ZEPLIN-III - veto system for various concentrations of gadolinium loading (by weight). Upper panel: the efficiency for the veto to tag events that based on ZEPLIN-III data alone could have been misidentified as WIMPs. Lower panel: For tagged events, the mean time delay in microseconds between signals seen in ZEPLIN-III and the veto.

The gadolinium is suspended in epoxy that is located in $2 \mathrm{~mm}$ wide, $10 \mathrm{~mm}$ pitch slots that cut through the full depth of the polypropylene. This choice has partly been driven by mechanical considerations, as narrower slots with a higher pitch would significantly increase the cost of machining. In Figures 17] and 18 the Monte Carlo simulation has been used to estimate the tagging efficiency as a function of slot width and slot pitch for a fixed average loading throughout the polypropylene of $0.5 \%$. Clearly, if the slot pitch increases beyond about $10 \mathrm{~mm}$, the efficiency of the veto in tagging coincident events within ZEPLIN-III begins to drop significantly. This 
is because, at this scale, neutrons moderating and thermalising within the polypropylene have a trajectory that sometimes never crosses a slot, and thus never provides an opportunity for capture on the gadolinium loading. The width of the slots has less effect, and thus a value amenable to construction can be chosen that minimises cost and is optimal for the epoxy to hold the gadolinium in suspension.

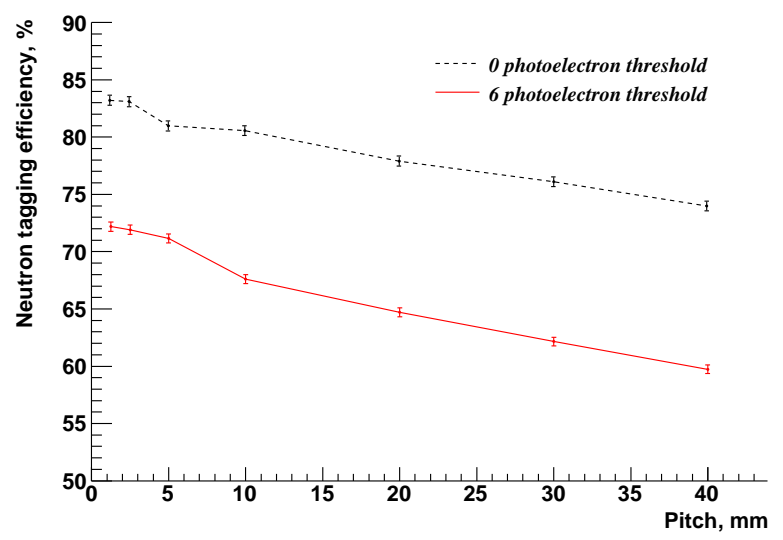

FIG. 17: Monte Carlo simulation of the efficiency of the veto in rejecting events that, based on ZEPLIN-III data alone, could have been identified as WIMPs, as a function of the pitch of the Gd-loaded epoxy filled slots. The width of the slots, $2 \mathrm{~mm}$, and the overall loading fraction of $\mathrm{Gd}, 0.5 \%$ by weight, is maintained in all cases.

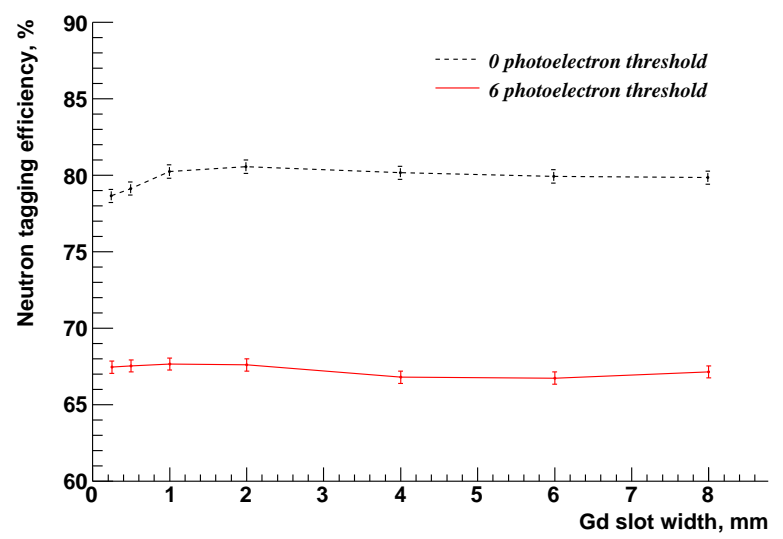

FIG. 18: Monte Carlo simulation of the efficiency of the veto in rejecting events that, based on ZEPLIN-III data alone, could have been identified as WIMPs, as a function of the width of the Gd-loaded epoxy filled slots. The pitch of the slots, $10 \mathrm{~mm}$, and the overall loading fraction of $\mathrm{Gd}, 0.5 \%$ by weight, is maintained in all cases.

\section{Predicted event rates}

The large volume of plastic scintillator used in the veto, combined with a low threshold, has the potential to create an extremely high event rate compatible neither with the data acquisition hardware nor the coincidence methodology to be used. The thorough approach to control and evaluate the radioactivity content of items used, coupled to the development of a detailed Monte Carlo simulation benchmarked against known performance, allows the absolute event rates to be estimated. The dominant rate comes from $\gamma$-rays, although it is the neutron background generated by the veto that is of fundamental importance to ZEPLIN-III. Excluding any contributions from the veto, the net nuclear recoil background in the second science run of the ZEPLIN-III experiment is expected to be $\simeq 0.4$ events per year. This is reduced to $\simeq 0.14$ events per year for a $65 \%$ veto tagging efficiency. For each contribution of background from the veto the complete Monte Carlo was performed, with the neutrons and $\gamma$-rays distributed in energy and location as appropriate. Energy depositions in the veto and in ZEPLINIII were recorded. The total expected event rate could then be calculated by summing the contributions. The net single scatter contribution to the background in the ZEPLIN-III acceptance region, assuming no rejection through vetoing the events, is 0.02 nuclear recoils per year. The nuclear recoil rate drops to 0.007 events per year when a conservative self-vetoing efficiency of $65 \%$ is applied. The veto is predicted to contribute $<1000$ electron recoils per year from $\gamma$-ray emission within the ZEPLIN-III fiducial volume, in the energy range of 2-16 keVee.

Considering the veto as a stand-alone instrument, Figure 19 shows the raw event rate that is expected in the veto as a function of the number of photoelectrons observed in a single plastic scintillator module. This shows, for example, that if a veto trigger requirement were imposed such that events were recorded if any single module recorded six or more photoelectrons, then a total event rate of $70 \mathrm{~Hz}$ would result.

We may consider how the background radioactivities will impact the veto when operating in coincidence with ZEPLIN-III by calculating the 'accidental coincidences rate'. This is the probability of any recorded timeline having an uncorrelated background-radioactivity caused signal within it. For the usual mode of slave operation, with the veto triggered to record for $320 \mu \mathrm{s}$ by events from ZEPLIN-III at an estimated rate of $1 \mathrm{~Hz}$, the accidental coincidences rate is indicated by the right hand axis in Figure 19. For nominal operational parameters the rate is less than $2 \%$.

\section{E. Impact of gaps in shielding}

The veto instrument consists of many modular pieces mechanically held together. As described in section II, the 


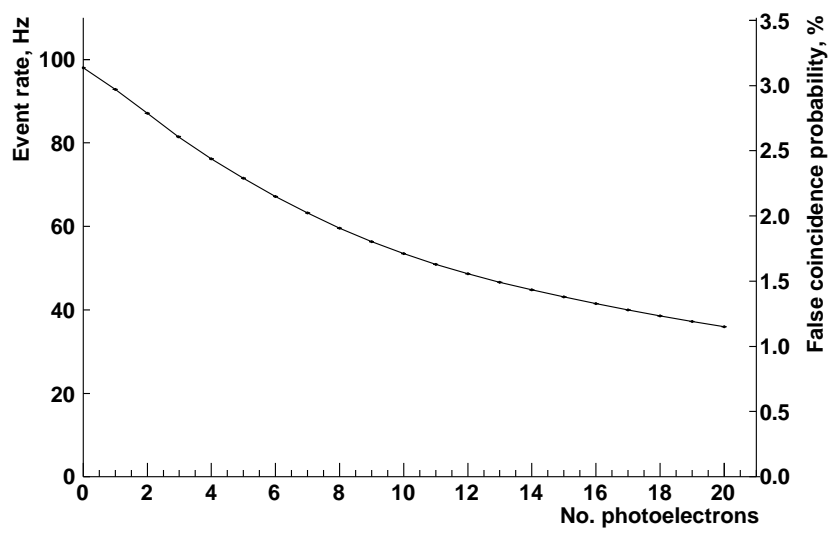

FIG. 19: Predicted event rate of energy depositions in the veto, as a function of signal size, originating from $\gamma$-ray emission of the measured activities of components. Statistical errors, although present, are too small to be seen clearly on this scale. Also shown is the probability for events seen in the veto following $\gamma$-ray emission from components being misidentified as coincident events with ZEPLIN-III.

plastic scintillators are deliberately offset relative to the polypropylene modules to prevent line-of-sight gaps that would compromise the shielding. There is, however, still a significant chance of introducing small gaps during construction and assembly. The Monte Carlo simulation has been employed to estimate the impact of such gaps occurring between the active modules, both in terms of degrading the shielding from external sources of background, and in terms of lowering the ability of the veto to tag coincident nuclear recoil events.

The simulation was performed for two distinct cases: when gaps existed between both active and passive modules, and when gaps existed between only active modules (in which case the passive modules were assumed to be completely closed). In the former case a direct path is formed for radiation that has penetrated the external lead shield to reach ZEPLIN-III. Simulation of a three year exposure that included gaps of $1 \mathrm{~mm}$ width, in excess of engineering constraints, showed no statistically significant increase in the ZEPLIN-III active volume single scatter rate. This is consistent with previous simulations performed to explore the impact of shielding due to the existing holes needed for pipework and cabling. Moreover, the veto tagging efficiency remains unaffected until the gaps exceed a width of $1 \mathrm{~cm}$. This is because a neutron, having originally entered the shielding or been internally generated, is unlikely to interact with ZEPLIN-III and exit the shielding through a gap again. In the latter sets of simulations, in which gaps between adjacent active scintillator modules were introduced assuming a continuous inner Gd loaded polypropylene barrel, it was found that the efficiency for tagging coincident veto - ZEPLIN-III nuclear recoil events begins to be significantly reduced for gaps of widths greater than $2 \mathrm{~cm}$, essentially due to less coverage for $\gamma$-ray detection following neutron capture. The neutron flux seen by ZEPLIN-III from external background is unaffected until the gap exceeds $1 \mathrm{~cm}$ between scintillator modules.

\section{PRESENT STATUS}

All veto components have been shipped underground to the Boulby Laboratory with active and passive modules coupled in preparation for installation. The full veto array, complete with data acquisition and synchronisation hardware, LED calibration systems and data reduction software has been successfully assembled within the $\mathrm{Pb}$ castle. This was done in the absence of ZEPLIN-III to test systems prior to final installation. Neutron and $\gamma$ ray source exposures as well as background runs have been performed and data recorded for in situ calibration and characterisation of the detector. The veto has since been dismantled to allow access to ZEPLIN-III. It will be installed following the completion of the upgrade to the ZEPLIN-III photomultiplier array. Performance results of the veto will be presented following commencement of the second science run of the ZEPLIN-III experiment.

\section{SUMMARY}

The second science run of the ZEPLIN-III project will feature two main upgrades: lower background photomultiplier tubes and the use of an active veto. Here, the design of the veto has been presented, together with details of the radiological content and performance of the components to be used. Detailed GEANT4 Monte Carlo simulations have been used to aid in the characterisation of the veto, and to estimate its overall performance. In addition to providing valuable diagnostic information, it is expected that the veto will be able to reject over $65 \%$ of neutrons, and over $15 \%$ of $\gamma$-rays from background radioactivities, whilst contributing negligibly to the ZEPLIN-III acceptance region background, expected to be $\simeq 0.4$ events per year. The veto will reduce this background to less than $\simeq 0.14$ events per year. This is a significant factor in the event of a non-zero observation. The veto has been fully assembled and systems integrated, and will be installed around ZEPLIN-III shortly for the commencement of the second science run as this instrument probes yet greater sensitivity.

\section{Acknowledgments}

The UK groups acknowledge the support of the Science \& Technology Facilities Council (STFC) for the ZEPLIN-III project and for maintenance and operation of the underground Palmer laboratory which is hosted by Cleveland Potash Ltd (CPL) at Boulby 
Mine, near Whitby on the North-East coast of England. The project would not be possible without the co-operation of the management and staff of CPL. We also acknowledge support from a Joint International Project award, held at ITEP and ICL, from the Russian Foundation of Basic Research (08-02-91851 $\mathrm{KO}$ a) and the Royal Society. LIP-Coimbra acknowledges financial support from Fundação para a Ciência e Tecnologia (FCT) through the project-grants
CERN/FP/83501/2008 and CERN/FP/109320/2009, as well as the postdoctoral grants SFRH/BPD/27054/2006 and SFRH/BPD/47320/2008. This work was supported in part by SC Rosatom; by Russian Grant SS-1329.2008.2 and by the Russian Ministry of Education and Science contract 02.740.11.0239. The University of Edinburgh is a charitable body, registered in Scotland, with the registration number SC005336.
[1] D. Yu. Akimov et al., Astroparticle Phys. 27 (2007) 4660.

[2] H. M. Araujo et al., Astroparticle Phys. 26 (2006) 140153.

[3] R. J. Gaitskell, Ann. Rev. Nucl. Part. Sci. 54 (2004)315.

[4] B. A. Dolgoshein, V. N. Lebedenko, B. U. Rodinov, JETP Lett. 11 (1970) 513.

[5] D. B. Cline et al. Astropart. Phys. 12 (2000) 373.

[6] S. Kubota et al., Phys. Rev. B 20 (8) (1979) 3486-3496.

[7] A. Hitachi et al., Phys. Rev. B 27 (9) (1979) 5279-5285.

[8] G. J. Davies et al., Phys. Lett. B 320 (1994) 395.

[9] V. Lebedenko et al., Phys. Rev. D., 80 (2009) 052010.

[10] V. Lebedenko et al., Phys. Rev. Lett., 103 (15) (2009) 151302.

[11] hepwww.rl.ac.uk/ukdmc/Radioactivity/Index.html

[12] Rutherford Appleton Laboratories Type 71 Mix Epoxy: www.rl.ac.uk

[13] M.B. Chadwick et al., At. Nucl. Data Tables 107 (2006) 2931-3118.

[14] H. M. Araujo et al, Astroparticle Physics 29 (2008) 471481.

[15] A. Lindote et al., Astroparticle Physics 31 (2009) 368-
375.

[16] A. Lindote, internal collaboration report (2008).

[17] S. Agostinelli et al. Nucl. Instru. Meth. A 506 (2003) 250-303.

[18] Amcrys-H Corp, Khakov, Ukraine: www.amcrys-h.com

[19] Amcrys-H Technical Note D13-2005-1111 UPS923A.

[20] 3M International Corp: www.3M.com/Vikuiti

[21] Plastomer Technologies: www.plastomertech.com

[22] Ensinger Corp: www.professionalplastics.com

[23] E. Barnes, PhD Thesis (2010), University of Edinburgh.

[24] J. B. Birks, J. Phys. B1 (1968) 946-957.

[25] E. N. Matveeva et al., Nucl. Instru. Meth. 179 (1981) 277-281.

[26] H. Klein et al. Nucl. Instru. Meth. A 476 (2002) 132-142.

[27] G. J. Alner et al., Astroparticle Phys. 28 (2007) 287.

[28] G. J. Alner et al., Phys. Lett. B 653 (2007) 161.

[29] W.B. Wilson et al., SOURCES-4C: A Code for Calculating $(\alpha, n)$ Spontaneous Fission and Delayed Neutron Sources and Spectra, American Nuclei Society/Radiation Protection and Shielding Division, 2002. 